Pacific Journal of Mathematics

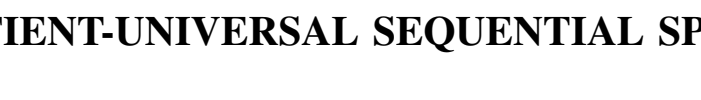




\title{
QUOTIENT-UNIVERSAL SEQUENTIAL SPACES
}

\author{
R. Sirois-Dumais AND S. Willard
}

We produce $2^{c}$ mutually nonhomeomorphic countable sequential spaces. These are used

(1) to answer in the negative the following question of Michael and Stone [4]: is every regular $T_{1}$-space which is a quotient of some separable metric space and a continuous image of the space $P$ of irrationals a quotient of $P$ ?

(2) to characterize $c$ (with or without the continuum hypothesis) as the smallest cardinal $\kappa$ with the property that a metric space of cardinality $\kappa$ exists of which every sequential space of cardinality $\leqq \kappa$ is a quotient.

1. Introduction. We let $Q$ denote the space of rationals, $\mathbf{P}$ the space of irrationals, $\mathbf{R}$ the real line, and $\mathbf{c}$ the cardinality of $\mathbf{R}$. For any set $X$, the cardinality of $X$ is denoted $|X|$.

We begin with the basic construction, which will be applied in the sequel in two different directions. Denote by $Y$ the set $[Q \times$ $(Q-\{0\})] \cup\{\infty\}$ and, for $E \subseteq \mathbf{R}$, denote by $\tau_{E}$ the quotient topology induced on $Y$ by the obvious map from the subspace $[Q \times(Q-\{0\})] \cup$ $(E \times\{0\})$ of $\mathbf{R} \times \mathbf{R}$. The set $Y$ endowed with the topology $\tau_{E}$ will be denoted $Y_{E}$. Note that $Y_{E}$ is a countable, regular, $T_{1}$-space which is, by construction, the quotient of a separable metric space. (Thus, see [3], $Y_{E}$ is both an $\boldsymbol{N}_{0}$-space and a $k$-space.)

2. Quotients of $\mathbf{P}$. In [4], Michael and Stone establish that every metrizable continuous image of $\mathbf{P}$ is a quotient of $\mathbf{P}$. The question is raised there whether this result can be extended to nonmetrizable images of $\mathbf{P}$, that is, whether a regular $T_{1}$-space which is at the same time a quotient of some separable metric space and a continuous image of $\mathbf{P}$ must be a quotient of $\mathbf{P}$. The construction of $\S 1$ provides the negative answer. To see this, first note that the countable discrete space (hence, every countable space) is a continuous image of $\mathbf{P}$ (collapse each interval $(n, n+1)$ to a point). It follows that each space $Y_{E}$ is a regular $T_{1}$-space which is a continuous image of $\mathbf{P}$ and a quotient of some separable metric space. But:

\section{THEOREM. Not every space $Y_{E}$ is a quotient of $\mathbf{P}$.}

Proof. If $E$ and $F$ are distinct subsets of $\mathbf{R}$, the topologies $\tau_{E}$ and $\tau_{F}$ on $Y$ are different, one containing a set containing $\infty$ which does not belong to the other. 
Now let $S$ be the set of all surjections $f: \boldsymbol{P} \rightarrow Y$ such that each $f^{-1}(y), y \in Y$, is closed in $\mathbf{P}$, and let $\Phi$ be the set of all $\phi: Y \rightarrow 2^{\mathbf{P}}$, where $2^{\mathbf{P}}$ denotes the collection of closed subsets of $\mathbf{P}$. Then $f \rightarrow f^{-1}$ is a one-one map from $S$ into $\Phi$; since $|\Phi|=\mathbf{c}^{\aleph_{0}}=\mathbf{c}$, we have $|S| \leqq c$. Let $J$ be the set of all $T_{1}$ topologies $\tau$ on $Y$ such that $(Y, \tau)$ is a quotient image of $\mathbf{P}$. Then each $\tau \in J$ is generated by some $f \in S$, so $|J| \leqq \mathbf{c}$. Since $\left|\left\{\tau_{E} \mid E \subseteq \mathbf{R}\right\}\right|=2^{\mathrm{c}}$, and since each $\tau_{E}$ is $T_{1}$, it follows that $\left(Y, \tau_{E}\right)$ is not a quotient of $\mathbf{P}$ for some $E \subseteq \mathbf{R}$.

Notes. (1) From the above, it is easily seen that there are $2^{c}$ nonhomeomorphic spaces $Y_{E}$, at most $\mathbf{c}$ of which can be quotients of $\mathbf{P}$. This result can be sharpened, with some difficulty. In fact, $Y_{E}$ is $a$ quotient of $\mathbf{P}$ iff $E$ is an analytic subset of $\mathbf{R}$.

(2) If, in the construction of $Y$, the set $Q \times(Q-\{0\})$ is replaced by a discrete space, say $\{(k / n, 1 / n) \mid k, n \in \mathbf{N}\}$, the spaces $Y_{E}$ which result still work, and have now the additional property that each has only one nonisolated point.

3. Quotient-universal sequential spaces. Let $\kappa$ be an infinite cardinal and let $S(\kappa)$ denote the collection of all sequential spaces of cardinality $\leqq \kappa$. A sequential space $S$ is quotient-universal ${ }^{*}$ for $S(\kappa)$ if $S \in S(\kappa)$ and every $T \in S(\kappa)$ is a quotient of $S$. We are particularly interested in the existence of metrizable quotient-universal spaces for $S(\kappa)$.

Whenever $\kappa^{\aleph_{0}}=\kappa$, the disjoint union of $\kappa$ copies of the converging sequence will serve as a metrizable quotient-universal space for $S(\kappa)$. In particular, there is a metrizable quotient-universal space for $S(c)$. In this section, we use the construction of $\S 1$ to demonstrate that, whether or not the continuum hypothesis is true, $\mathbf{c}$ is the smallest cardinal for which this is true. In fact, we exhibit a countable sequential space which is not a quotient of any metric space of cardinality $<\mathbf{c}$.

LEMMA. There exists a subset $E$ of $\mathbf{R}$ with $|E|=\mathbf{c}$ which contains no uncountable closed subset of $\mathbf{R}$.

Proof. Let $\left\{C_{\alpha} \mid \alpha<\mathbf{c}\right\}$ be a transfinite enumeration of the $\mathbf{c}$ uncountable closed subsets of R. Pick $p_{0}$ and $q_{0}$ in $C_{0}$ with $p_{0} \neq q_{0}$. If $p_{\alpha}$ and $q_{\alpha}$ have been chosen in $C_{\alpha}$ for $\alpha<\beta$ so that all $p_{\alpha}$ and $q_{\alpha}$ are distinct, choose $p_{\beta}$ and $q_{\beta}$ in $C_{\beta}$ so that $p_{\beta} \neq q_{\beta}$ and $p_{\beta}, q_{\beta}$ are distinct from all $p_{\alpha}, q_{\alpha}$ for $\alpha<\beta$. This is possible since any uncountable closed subset of $\mathbf{R}$ has cardinal $\mathbf{c}$ so that $C_{\beta}-\left\{p_{\alpha}, q_{\alpha} \mid \alpha<\beta\right\} \neq \phi$.

* The term "universal" has been preempted by those who study spaces with a given property $P$ which contain as subspaces every space (of appropriate cardinality or weight) having property $P$. See, for example, [2], [5] and [6]. 
Let $E=\left\{p_{\alpha} \mid \alpha<\mathbf{c}\right\}$. Then $|E|=\mathbf{c}$ and $E$ contains no uncountable closed subset of $\mathbf{R}$ since $q_{\alpha} \in C_{\alpha}-E$ for each $\alpha$.

Let $E \subseteq \mathbf{R}$ be the set of the lemma. Let $M_{E}$ denote the subspace $[Q \times(Q-\{0\})] \cup(E \times\{0\})$ of $\mathbf{R} \times \mathbf{R}$. Recall that $Y_{E}$ is the quotient of $M_{E}$ obtained by collapsing $E \times\{0\}$ to a single point $e$. Let $q: M_{E} \rightarrow Y_{E}$ be the quotient map.

$Y_{E}$ is a countable sequential space, but:

THEOREM. $\quad Y_{E}$ is not the quotient of any metric space of cardinality $<\mathbf{c}$.

Proof. Suppose there is a quotient map $f$ of $S$ onto $Y_{E}$, where $S$ is a metric space and $|S|=\kappa<\mathbf{c}$. For each $p \in E$, let $\sigma_{p}=\left(x_{p 1}, x_{p 2}, \cdots\right)$ be a sequence in $Q \times(Q-\{0\})$ such that

$$
\left|x_{p n}-(p, 0)\right| \leqq \min \left\{\frac{1}{n},\left|x_{p n-1}-(p, 0)\right|\right\} .
$$

Recall that $q$ denotes the quotient map of $M_{E}$ onto $Y_{E}$. For each $n$, let

$$
z_{p n}=q\left(x_{p n}\right)
$$

and denote by $\eta_{p}$ the sequence $\left(z_{p 1}, z_{p 2}, \cdots\right)$ in $Y_{E}$. Now $\eta_{p} \rightarrow e$. Hence, since $f$ is a hereditary quotient map, there exists some $b_{p} \in f^{-1}(e)$ and a sequence $\sigma_{p}=\left(s_{p 1}, s_{p 2}, \cdots\right)$ in $S-f^{-1}(e)$ such that $\sigma_{p} \rightarrow b_{p}$ and $f\left(\sigma_{p}\right)=\eta_{p}$. Let

$$
f^{-1}(e)=\left\{x_{\alpha} \mid \alpha<\kappa\right\}
$$

and, for $\alpha<\kappa$, let

$$
A_{\alpha}=\left\{p \in E \mid b_{p}=x_{\alpha}\right\}
$$

We claim some $A_{\alpha}$ must contain a sequence $\left(p_{t}\right)$ converging to some element of $\mathbf{R}-E$. For otherwise $C 1_{\mathbf{R}}\left(A_{\alpha}\right) \subset E$ for each $\alpha<\kappa$, whence $E$ is the union of fewer than closed sets. But since $|E|=\mathbf{c}$, one of these would be an uncountable closed set in $E$, contradicting the construction of $E$.

Without loss of generality, say $A_{1}$ contains a sequence $\left(p_{t}\right)$ which is closed and discrete in $E$. Then the sequence $\eta_{p_{t}}=\left(z_{p_{1} 1}, z_{p, 2}, \cdots\right)$ converges to $e$, for each $i$, and the sequence $\delta_{p_{t}}=\left(s_{p_{1}}, s_{p_{1},}, \cdots\right)$ converges to $x_{1}$, for each $i$. A diagonal sequence $\left(s_{p_{1} n_{1}}, s_{p_{2} n_{2}}, \cdots\right)$ with $n_{k} \geqq k$ for each $k$ will then converge to $x_{1}$. Then $\left(z_{p_{1} n_{1}}, z_{p_{2} n_{2}}, \cdots\right)$ converges to $e$. Hence $\left(x_{p_{1} n_{1}}, x_{p_{2} n_{2}}, \cdots\right)$ must have a cluster point in $M_{E}$. 
But $\left|x_{p_{k} n_{k}}-\left(p_{k}, 0\right)\right| \leqq\left|x_{p_{k} k}-\left(p_{k}, 0\right)\right| \leqq 1 / k$, so any cluster point of $\left(x_{p_{1} n_{1}}, x_{p_{2} n_{2}}, \cdots\right)$ in $M_{E}$ would be a cluster point of $\left(\left(p_{1}, 0\right),\left(p_{2}, 0\right), \cdots\right)$, which is impossible by choice of the $p_{1}$.

We conclude with some observations on extension of the result above.

(1) As noted in $\S 2$, there are $2^{c}$ mutually nonhomeomorphic spaces $Y_{E}$. Since there are at most $\mathbf{c}$ quotients of any single countable sequential space, there can exist no quotient-universal space (metrizable or not) for $S\left(\boldsymbol{N}_{0}\right)$. It is at least consistent with the usual (ZermeloFraenkel) axioms for set theory (with Choice) that this result extends to all cardinals $\kappa<\mathbf{c}$, for Martin's axiom entails $2^{\kappa}<2^{\mathbf{c}}$ for $\kappa<\mathbf{c}$.

(2) Let $M(\kappa)$ denote the collection of metrizable spaces of cardinal $\leqq \kappa$. The space $Q$ of rationals is a (metrizable) quotientuniversal space for $M\left(\boldsymbol{\aleph}_{0}\right)$, while the disjoint union of $\mathbf{c}$ copies of the converging sequence is a quotient-universal space for $M(\mathbf{c})$. For cardinals $\kappa$ between $\boldsymbol{N}_{0}$ and $\mathrm{c}$ little is known. Baumgartner ([1]) has shown that it is consistent with Zermelo-Fraenkel set theory with choice that all $\boldsymbol{N}_{1}$-dense subsets of $\mathbf{R}$ are order-isomorphic. (A subset $A$ of $\mathbf{R}$ is $\boldsymbol{\aleph}_{1}$-dense if whenever $a<b$ in $\mathbf{R},(a, b) \cap A$ has cardinal $\left.\boldsymbol{\aleph}_{1}\right)$ If this is the case, then every separable metric space $\boldsymbol{M}$ of cardinal $\leqq \boldsymbol{N}_{1}$ is a quotient of the unique $\boldsymbol{N}_{1}$-dense subset $D$ of $\mathbf{R}$. For $M$ is a quotient of $M \times D$, while ([7], Theorem 76) $M \times D$ is homeomorphic to a subset of $\mathbf{R}$ and hence, by Baumgartner's result, to $D$.

\section{REFERENCES}

1. James E. Baumgartner, All $\boldsymbol{\aleph}_{1}$-dense sets of reals can be isomorphic, Fund. Math., 79 (1973), 103-106.

2. Jonsson, Universal Relational Systems, Math. Scand., 4 (1956), 193-208.

3. E. Michael, $\boldsymbol{N}_{(1)}$-spaces, J. Math. Mech., 15 (1966), 983-1002.

4. E. Michael and A. H. Stone, Quotients of the space of irrationals, Pacific J. Math., 28 (1969), 629-633.

5. R. Pol, There is no universal totally disconnected space, Fund. Math., 79 (1973), 265-267.

6. W. Sierpinski, Sur les espaces métriques universels, Fund. Math., 33 (1945), 123-136.

7. - General Topology, 2nd ed., U. of Toronto Press, 1956 (translated by C. C. Krieger).

Received March 12, 1976 and in revised form May 12, 1976.

UNIVERSity of AlbERTA 



\section{Pacific Journal of Mathematics}

\section{Vol. 66, No. $1 \quad$ November, 1976}

Helen Elizabeth. Adams, Factorization-prime ideals in integral domains ............ Patrick Robert Ahern and Robert Bruce Schneider, The boundary behavior of Henkin's kernel.

Daniel D. Anderson, Jacob R. Matijevic and Warren Douglas Nichols, The Krull

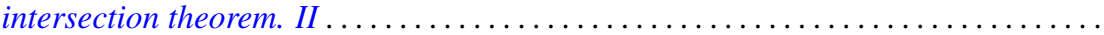

Efraim Pacillas Armendariz, On semiprime P.I.-algebras over commutative regular

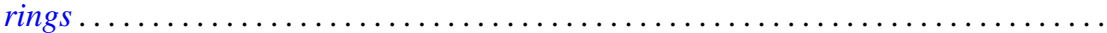

Robert H. Bird and Charles John Parry, Integral bases for bicyclic biquadratic fields

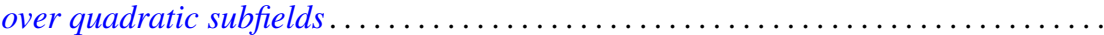

Tae Ho Choe and Young Hee Hong, Extensions of completely regular ordered

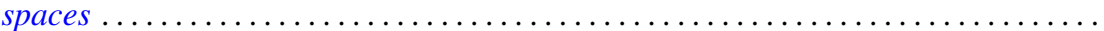

John Dauns, Generalized monoform and quasi injective modules ...............

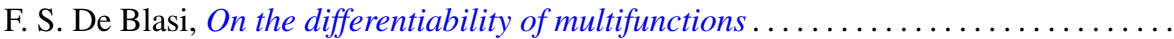

Paul M. Eakin, Jr. and Avinash Madhav Sathaye, R-endomorphisms of $R[[X]]$ are

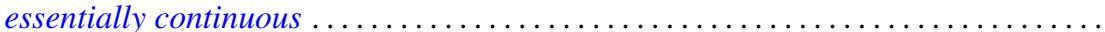

Larry Quin Eifler, Open mapping theorems for probability measures on metric

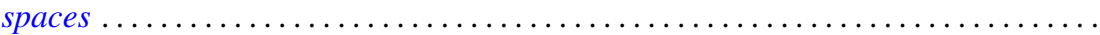

Garret J. Etgen and James Pawlowski, Oscillation criteria for second order self adjoint

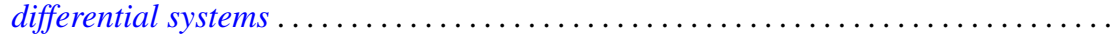

Ronald Fintushel, Local $S^{1}$ actions on 3-manifolds .

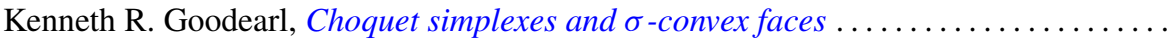

John R. Graef, Some nonoscillation criteria for higher order nonlinear differential

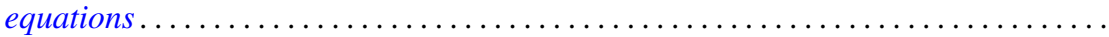

Charles Henry Heiberg, Norms of powers of absolutely convergent Fourier series: an example.

Les Andrew Karlovitz, Existence of fixed points of nonexpansive mappings in a space

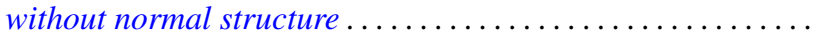

Gangaram S. Ladde, Systems of functional differential inequalities and functional

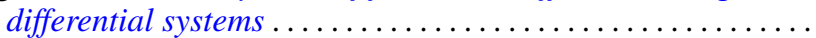

Joseph Michael Lambert, Conditions for simultaneous approximation and interpolation with norm preservation in $C[a, b]$.

Ernest Paul Lane, Insertion of a continuous function.

Robert F. Lax, Weierstrass points of products of Riemann surfaces .

Dan McCord, An estimate of the Nielsen number and an example concerning the Lefschetz fixed point theorem...

Paul Milnes and John Sydney Pym, Counterexample in the theory of continuous functions on topological groups...

Peter Johanna I. M. De Paepe, Homomorphism spaces of algebras of holomorphic functions

Judith Ann Palagallo, A representation of additive functionals on $L^{p}$-spaces,

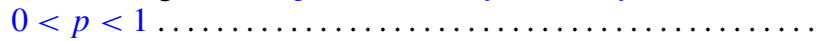

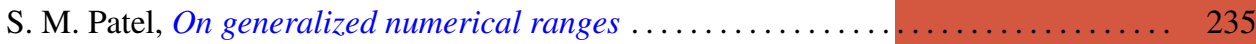

Thomas Thornton Read, A limit-point criterion for expressions with oscillatory

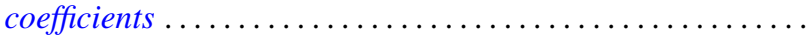

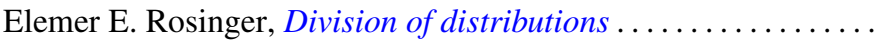

Peter S. Shoenfeld, Highly proximal and generalized almost finite

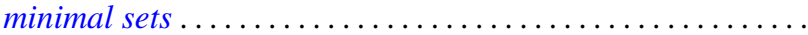

R. Sirois-Dumais and Stephen Willard, Quotient-universal sequential spaces

Robert Charles Thompson, Convex and concave functions of singular values of matrix sums....

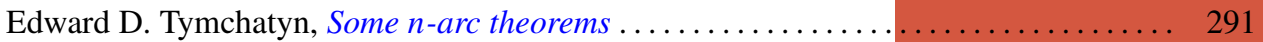

